\title{
Author Correction: Flexible filaments buckle into helicoidal shapes in strong compressional flows
}

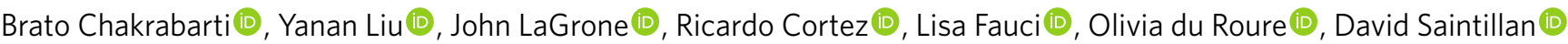
and Anke Lindner (D)

Correction to: Nature Physics https://doi.org/10.1038/s41567-020-0843-7, published online 30 March 2020

In the version of this Article initially published, in the section titled "Helical shapes stem from interacting planar modes," seventh paragraph, the text reading "Solutions of this system exhibit amplitude saturation following an initial exponential growth regime, and in sufficiently strong flows always produce 3D helical conformations consistent with observations" has been replaced with the text "Solutions of this system always produce 3D helical conformations in sufficiently strong flows, consistent with observations."

The authors note that the weakly nonlinear theory does not lead to amplitude saturation as seen in fully nonlinear simulations. However, the main prediction that the formation of helical shapes results from the nonlinear coupling of planar modes remains unchanged.

The associated Supplementary Information file and Supplementary Video 9 have been replaced to reflect this update as well.

The original Article has been corrected in the online version of the paper.

Published online: 27 August 2021

https://doi.org/10.1038/s41567-021-01372-3

(C) The Author(s), under exclusive licence to Springer Nature Limited 2021 